\title{
Differential Contribution of Cortical and Subcortical Visual Pathways to the Implicit Processing of Emotional Faces: A tDCS Study
}

\author{
Roberto Cecere, ${ }^{1,2}$ Caterina Bertini, ${ }^{1,2}$ and Elisabetta Làdavas ${ }^{1,2}$ \\ ${ }^{1}$ Dipartimento di Psicologia, Università di Bologna, 40127 Bologna, Italy and ${ }^{2}$ CSRNC, Centro Studi e Ricerche in Neuroscienze Cognitive, Polo \\ Scientifico-Didattico di Cesena, 47023 Cesena, Italy
}

\begin{abstract}
The visual processing of emotional faces is subserved by both a cortical and a subcortical route. To investigate the specific contribution of these two functional pathways, two groups of neurologically healthy humans were tested using transcranial direct current stimulation (tDCS). In Experiment 1, participants received sham and active cathodal-inhibitory tDCS over the left occipital cortex, while, in control Experiment 2, participants received sham and active cathodal-inhibitory tDCS over the vertex, to exclude any unspecific effect of tDCS. After tDCS, participants performed a go/no-go task responding to happy or fearful target faces presented in the left visual field, while backwardly masked faces (emotionally congruent, incongruent, or neutral) were concurrently displayed in the right visual field. After both suppressing activity in the vertex (Experiment 2) and sham stimulation (Experiment 1 and 2) a reduction of reaction times was found for pairs of emotionally congruent stimuli. However, after suppressing the activity in the left occipital cortex, the congruency-dependent response facilitation disappeared, while a specific facilitative affect was evident when masked fearful faces were coupled with happy target faces. These results parallel the performances of hemianopic patients and suggest that when the occipital cortex is damaged or inhibited, and the visual processing for emotional faces is mainly dependent on the activation of the "low road" subcortical route, fearful faces represent the only visually processed stimuli capable of facilitating a behavioral response. This effect might reflect an adaptive mechanism implemented by the brain to quickly react to potential threats before their conscious identification.
\end{abstract}

\section{Introduction}

Converging evidence suggests that fear-related emotional signals are processed by two parallel pathways: a cortical "high road," from the thalamic lateral geniculate nucleus to the striate cortex and amygdala, for fine-grained, but slow evaluations of stimuli, and a subcortical "low road," encompassing the superior colliculus, thalamic pulvinar, and amygdala, for fast, but coarse analysis of potential threat (LeDoux, 1996).

The low road is reportedly involved in the implicit visual processing of emotional stimuli, both in healthy participants (Whalen et al., 1998; Morris et al., 1999) and in patients with visual field defects (Morris et al., 2001). In particular, studies on these patients suggest that unseen emotional stimuli in the blind field might be processed even in the absence of explicit awareness (de Gelder et al., 1999, 2001; Bertini et al., 2012).

However, the nature of this implicit process is still debated, and different results have been reported in studies on patients

\footnotetext{
Received July 18, 2012; revised Jan. 30, 2013; accepted Feb. 8, 2013.

Author contributions: R.C., C.B., and E.L. designed research; R.C. performed research; R.C. and C.B. analyzed data; R.C., C.B., and E.L. wrote the paper.

The authors declare no competing financial interests.

This research was supported by grants from the Ministero Istruzione Università e Ricerca (PRIN) to E.L. We are grateful to Neil Dundon for his help in editing this manuscript.

Correspondence should be addressed to Elisabetta Làdavas, Department of Psychology, University of Bologna, Viale Berti Pichat, 5-40127 Bologna, Italy. E-mail: elisabetta.ladavas@unibo.it.

DOI:10.1523/JNEUROSCI.3431-12.2013

Copyright $\odot 2013$ the authors $\quad 0270-6474 / 13 / 336469-07 \$ 15.00 / 0$
}

with visual field defects. Classical blindsight patients, tested with two-alternative forced choice tasks, reported above-chance discrimination of the emotional content of faces presented in the blind field (de Gelder et al., 1999) and reduced reaction times (RTs) to congruent pairs of sad or fearful faces, during a go/no-go task with redundant stimuli (de Gelder et al., 2001). Conversely, in a recent study on hemianopic patients with no residual discriminative abilities in their blind field, RTs were only facilitated by unseen fearful stimuli. In contrast to blindsight patients, no facilitation was evident in response to congruent pairs of emotional stimuli (Bertini et al., 2012).

This contrasting evidence might reflect different cognitive processes and raise the debate about the possible contribution of different neural networks.

In particular, the above-chance emotional discrimination and the congruency-dependent facilitative effect observed in blindsight patients might reflect a high-level cognitive process, requiring the contribution of both the low road and some spared occipital areas of the cortical high road. In contrast, the fearspecific facilitation reported in hemianopic patients with no discriminative abilities for unseen stimuli might reflect a low-level process, exclusively relying on the low road.

This study used cathodal-inhibitory transcranial direct current stimulation (tDCS) to modulate activity in healthy occipital cortex to infer the cortical contributions to implicit emotionalface processing. Following sham or active cathodal tDCS over their occipital cortex, healthy participants performed a go/no-go 
task with redundant stimuli, responding to seen emotional faces (fearful or happy) concurrently displayed with neutral, emotionally congruent, or emotionally incongruent masked faces (Experiment 1). To exclude any unspecific effect of tDCS, another group was tested with the same task, after sham or active cathodal tDCS over a control site, namely the vertex (Experiment 2). Following sham stimulation, faster responses for pairs of emotionally congruent stimuli are expected, in line with literature on healthy participants (Tamietto and de Gelder, 2008). In contrast, the suppression of activity of the occipital areas should disrupt the congruency-dependent facilitation and yield a specific reduction of RTs for unseen (masked) fearful faces, consistently with evidence on hemianopic patients (Bertini et al., 2012).

\section{Materials and Methods}

Participants. Twenty-six right-handed healthy volunteers (21 females; mean age: 24 years; range: $21-28$ years) participated in Experiment 1, while 24 ( 21 females; mean age: 24 years; range $21-31$ years) participated in Experiment 2. All participants gave their written consent and completed a $\mathrm{tDCS}$ safety screening form before taking part in the study, which was approved by the Departmental Ethics Committee.

Brain stimulation procedure. Brain stimulation has been proven to be effective in suppressing the occipital cortex excitability, interfering with visual processing (Bertini et al., 2010). tDCS is a noninvasive method for delivering weak polarizing electrical current to the human cortex, focally altering neural resting membrane potential and inducing prolonged changes in synaptic efficiency. More specifically, while anodal tDCS enhances cortical excitability, cathodal stimulation reduces it. In the visual domain, it has been shown that tDCS is able to modulate the amplitude of visual-evoked potentials (Antal et al., 2004a), modify the perception of phosphenes (Antal et al., 2003a,b), and affect contrast sensitivity (Antal et al., 2001) and motion detection (Antal et al., 2004b). In particular, cathodal tDCS has been proven to induce a significant decrease in static and dynamic contrast sensitivity (Antal et al., 2001) and elicit a threshold increase for static phosphenes (Antal et al., 2003a).

Before undergoing the behavioral task, participants received either active or sham tDCS over the left occipital cortex (Experiment 1) or the vertex (Experiment 2). In each experiment, the order of the tDCS sessions was counterbalanced and at least 1 week was allowed between sessions. A direct current of $2 \mathrm{~mA}$ intensity was delivered by a battery-driven, constant-current stimulator (ELDITH DC-Stimulator; Neuroconn), through a couple of rubber electrodes in $5 \times 7 \mathrm{~cm}$ saline-soaked sponges. The impedance was kept $\leq 5 \mathrm{~K} \Omega$ during the stimulation. In the active $\mathrm{tDCS}$ sessions, cathodal stimulation was applied for $900 \mathrm{~s}$ ( $15 \mathrm{~min}$; fade in-out duration: $30 \mathrm{~s}$ ). This duration has been shown to produce a $20 \mathrm{~min}$ aftereffect at $1 \mathrm{~mA}$ intensity (Antal et al., 2004a). In the sham sessions, the direct current was delivered for $30 \mathrm{~s}$ to reproduce the early sensations of the active stimulation, and then it was switched off.

To inhibit left occipital areas in Experiment 1, the cathodal electrode was placed over left O1 (according to the EEG 10/20 system), and the anodal electrode over $\mathrm{Cz}$. The $\mathrm{Oz}-\mathrm{Cz}$ montage has been proven to be the optimal stimulating electrode position to suppress activity in the visual cortex, compared with several other electrode montages, such as the left O1-right $\mathrm{O}_{2}$ montage or the Oz-left mastoid montage (Antal et al., 2004a). In Experiment 2, the position of the electrodes was reversed by placing the cathode over $\mathrm{Cz}$ and the anode over O1. Stimulation was delivered only to the left hemisphere to avoid right hemisphere suppression, since a wide range of evidence in the literature has reported the involvement of the right hemisphere in the emotional processing of faces (for review, see Adolphs, 2002).

Experimental task. Before each experimental session, participants underwent two training blocks of the behavioral go/no-go task (one with fearful and one with happy faces as targets). After the practice, participants received brain stimulation (active tDCS or sham tDCS) over O1 (Experiment 1) or $\mathrm{Cz}$ (Experiment 2) before undergoing a go/no-go task with redundant stimuli. The same task was used both in Experiments 1 and 2 and it was performed in $\sim 16$ min to stay within the $20 \mathrm{~min}$ tem- poral window of the tDCS aftereffect (Antal et al., 2004a). Each session, including training, brain stimulation, experimental task, and a final check of the masking procedure effectiveness, lasted 90 min overall.

During the go/no-go task, participants sat in front of a 17 inch LCD monitor (sampling rate $60 \mathrm{~Hz}$ ), at a distance of $57 \mathrm{~cm}$. Participants were asked to respond by pressing a button on a standard keyboard. Stimulus display and RTs were recorded and controlled by a computer running E-Prime software (E-Prime 1.1; Psychology Software Tools). Participants' eye movements were controlled on-line throughout the experiment by an infrared eye-tracking system (Eye-Track ASL-6000; sampling rate $60 \mathrm{~Hz})$. Trials with eye movements $(<1 \%$ in total) were marked on-line by the experimenter via a mouse button press and subsequently discarded from analyses.

Stimuli consisted of 18 gray scale photographs $\left(7.5^{\circ} \times 11^{\circ}\right)$ from the Pictures of Facial Affect set (Ekman and Friesen, 1976), featuring six different identities (three males) displaying a fearful, happy or neutral facial expression.

In each trial, target stimuli consisted of fearful or happy faces displayed for $200 \mathrm{~ms}$ in the left visual field (LVF), ipsilateral to the stimulated site. Concurrently, backwardly masked fearful, happy, or neutral faces were presented in the right visual field (RVF), contralateral to the stimulated site. The stimulus/mask compounds consisted of fearful, happy, or neutral faces presented for $33 \mathrm{~ms}$, immediately replaced by a mask ( $167 \mathrm{~ms})$, to prevent conscious perception. The mask consisted of a neutral face of different identity to the previous stimulus. Targets and stimulus/mask compounds were always presented bilaterally at $10^{\circ}$ to the left and to the right of the central fixation cross. The $33 \mathrm{~ms}$ stimulus onset asynchrony (SOA) used for the masking procedure was proven to be effective in preventing conscious perception by a preliminary pilot experiment, consistently with previous studies (Esteves and Ohman, 1993; Morris et al., 1998; Whalen et al., 1998; Etkin et al., 2004).

Each trial started with a blank screen with a central fixation cross (500 $\mathrm{ms}$ ), followed by the presentation of the targets and stimulus/mask compounds $(200 \mathrm{~ms})$ and a subsequent blank screen with a central fixation cross $(1000 \mathrm{~ms})$. Intertrial intervals ranged between 500 and $800 \mathrm{~ms}$.

While fixating at the central cross, participants were assigned to respond, with their right hand and as quickly and accurately as possible, to the target emotional face in the LVF (fearful or happy), paired with the masked face in the RVF (fearful, happy, or neutral; Fig. 1). In half of the blocks the target faces in the LVF were fearful faces and nontargets were happy faces, while in the other half the target/nontarget faces were reversed. As a result, there were six possible conditions of stimulus presentation: (1) target fearful face/ masked neutral face (Fn), (2) target fearful face/masked fearful face (Ff), (3) target fearful face/masked happy face (Fh), (4) target happy face/masked neutral face (Hn), (5) target happy face/masked happy face (Hh), and (6) target happy face/masked fearful face (Hf).

In each $\mathrm{tDCS}$ session, eight experimental blocks were run in a counterbalanced order. Each block consisted of 36 trials (18 valid trials, 6 repetitions per condition), resulting in 288 trials overall (144 valid trials, 24 repetitions per condition). At the end of each experimental session, a forced triple-choice task, also used in the pilot experiment to set the SOA parameters, was performed to check the effectiveness of the masking procedure (see below).

Masking procedure effectiveness analysis. After performing the behavioral go/no-go task, the effectiveness of the backward masking procedure in preventing conscious perception was checked in each participant, using a forced triple-choice task with the same masked stimuli used throughout the experiments. A single block of 144 trials (lasting $\sim 8 \mathrm{~min}$ ) was run, where participants were required to fixate on a central cross and to discriminate masked fearful, happy, and neutral faces, by pressing different keys on a standard keyboard. All stimuli were presented unilaterally in the RVF at $10^{\circ}$ eccentricity from the fixation cross. Stimulus display and SOA parameters were the same as in the experimental sessions. Sensitivity $\left(d^{\prime}\right)$ scores were computed for each participant and those with discriminative ability above the chance level were excluded from the statistical analysis ( 2 of 26 participants in Experiment 1 ; all $d^{\prime}>$ 0.7 , all $p s<0.03)$.

Since half of the trials in the task used to test the masking effectiveness fell inside the aftereffect window of cathodal/anodal tDCS over O1, we 
Target Unseen face Target Mask

a
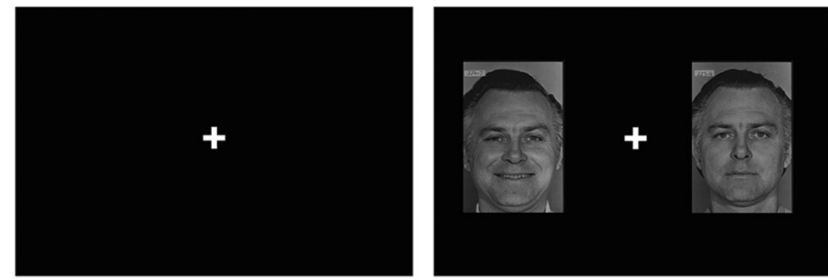

b
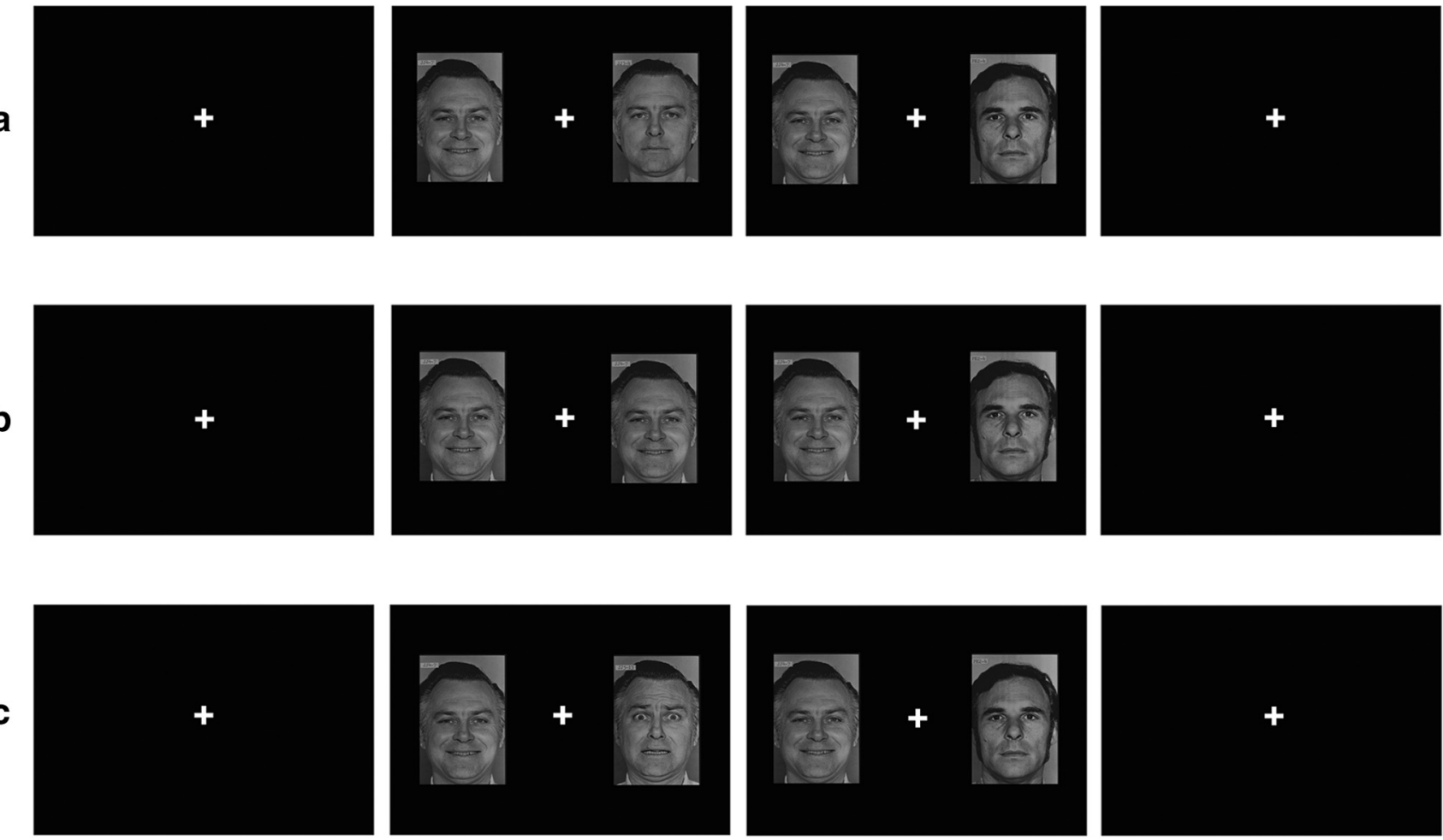

$500 \mathrm{~ms}$

$33 \mathrm{~ms}$

$167 \mathrm{~ms}$

$1000 \mathrm{~ms}$

Figure 1. Schematic representation of the experimental task. The figure depicts examples of neutral $(\boldsymbol{a})$, congruent $(\boldsymbol{b})$, and incongruent (c) trials, when the target is a happy face. In each trial, the target face was displayed for $200 \mathrm{~ms}$ in the LVF. Concurrently, backwardly masked neutral, emotionally congruent, or emotionally incongruent faces were presented in the RVF. The stimulus/ mask compounds consisted of fearful, happy, or neutral faces presented for $33 \mathrm{~ms}$, immediately replaced by a neutral face (167 ms), to prevent conscious perception.

performed an additional analysis to control for possible differential effects of cathodal and anodal stimulation on perceptual sensitivity (i.e., masking effectiveness). For each participant from the two groups receiving cathodal and anodal tDCS over O1, we split the 144-trial block into two parts ( 72 in-window trials and 72 out-window trials) and computed the $d^{\prime}$ in each of the four resulting conditions (cathodal group/inwindow, cathodal group/out-window and anodal group/in-window, anodal group/out-window). None of the participants had resulting $d^{\prime}$ values significantly above chance (all $p s>0.74$ ). $d^{\prime}$ values were entered into an ANOVA with time (in-window, out-window) as a withinparticipants factor and group (cathodal, anodal) as a betweenparticipants factor. Neither the main effects (all $F s<0.8$; all $p s>0.37$ ) nor the interaction $\left(F_{(1,46)}=0.09 ; p=0.76\right)$ was significant. Moreover, we directly compared $\mathrm{d}^{\prime}$ values between the in-windows of the cathodal and anodal tDCS groups. $d^{\prime}$ values were not significantly different between the two groups $(t=-0.31 ; p=0.99)$. In summary, analyses did not show any difference in sensitivity either between trials falling inside and outside the tDCS aftereffect window, or between cathodal and anodal tDCS.

Data analysis. To control for outliers, RTs exceeding 1.5 SDs above or below each participant's condition mean were removed from the analyses. The rejection rates resulting from this procedure were as follows: $11 \%$ of the trials in the $\mathrm{O} 1$ active cathodal, $\mathrm{O} 1$ sham and $\mathrm{Cz}$ active cathodal tDCS sessions; $10 \%$ of the trials in the Cz sham tDCS session. Statistical analyses were performed on RTs of correct responses; mean error rates were as follows: $5 \%( \pm 7 \%)$ in the $\mathrm{O} 1$ active cathodal, $4 \%$ $( \pm 6 \%)$ in the $\mathrm{O} 1 \mathrm{sham}, 3 \%( \pm 4 \%)$ in the $\mathrm{Cz}$ active cathodal, and $4 \%$ $( \pm 5 \%)$ in the $\mathrm{Cz}$ sham $\mathrm{tDCS}$ sessions.

All RTs from all of the experiments were entered in a general ANOVA with session (active cathodal tDCS, sham tDCS), target emotion (fear, happiness), and condition (neutral, congruent, incongruent) as within- participants factors and experiment (target area $\mathrm{O} 1$, target area $\mathrm{Cz}$ ) as a between-participants factor. The results revealed a significant session by condition by experiment interaction $\left(F_{(2,92)}=3.33 ; p=0.04\right)$. Therefore, Experiments 1 and 2 were separately analyzed, performing two ANOVAs with session (active cathodal tDCS, sham tDCS), target emotion (fear, happiness), and condition (neutral, congruent, incongruent) as within-participants factors. All resulting significant effects and interactions were further analyzed using the Duncan post hoc test.

\section{Results}

\section{Experiment 1: sham versus active cathodal tDCS over the} occipital cortex

In the main experiment participants received, in two separate sessions, either active cathodal or sham tDCS over left occipital areas before the execution of a behavioral go/no-go task. RTs were recorded in response to target emotional faces (fearful of happy) presented in the LVF, ipsilaterally to the stimulated site, which were coupled with masked emotional faces (emotionally congruent, emotionally incongruent, or neutral) presented in the RVF, contralaterally to the stimulated site.

The results of the $2 \times 2 \times 3$ ANOVA on RTs with session (active cathodal tDCS over left O1, sham tDCS), target emotion (fear, happiness), and condition (neutral, congruent, incongruent) as within-participants factors revealed a significant threeway interaction $\left(F_{(2,46)}=3.59, p=0.036\right)$. To further investigate the three-way interaction, two separate analysis for the cathodal tDCS over $\mathrm{O} 1$ and the sham tDCS sessions were carried out, by means of two $2 \times 3$ ANOVAs, with target emotion (fear, happi- 


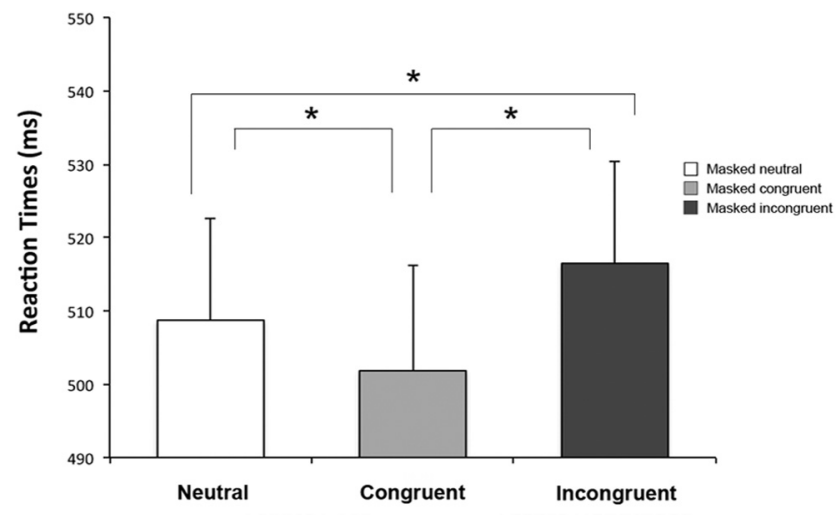

Figure 2. Experiment 1: Sham tDCS over 01. Mean RTs are reported for the neutral condition (Fn, Hn), the congruent condition (Ff, Hh), and the incongruent condition (Fh, Hf). Error bars indicate SEM. Asterisks indicate significantly faster RTs in the congruent condition than in the neutral and incongruent conditions (all $p s \leq 0.04$ ) and significantly slower RTs in the incongruent than in the neutral condition $(p=0.04)$.

ness) and condition (congruent, incongruent, neutral) as withinparticipants factors.

When sham stimulation was delivered, the target emotion by condition ANOVA showed a significant main effect for the condition factor $\left(F_{(2,46)}=8.69, p=0.0006\right)$. As revealed by the post hoc comparisons, RTs were significantly faster in the congruent conditions (Ff and Hh, mean RT $502 \mathrm{~ms}$ ) compared with both the neutral conditions (Fn and Hn, mean RT $507 \mathrm{~ms}, p=0.04$ ) and the incongruent conditions (Fh and Hf, mean RT $513 \mathrm{~ms}, p=$ $0.0002)$. Moreover, RTs in the incongruent conditions were slower compared with the neutral condition ( $p=0.04$; Fig. 2 ). The main effect of target emotion and the target emotion by condition interaction were not significant $\left(F_{(1,23)}=0.26, p=\right.$ $0.61 ; F_{(2,46)}=0.74, p=0.48$, respectively). To sum up, when participants received sham stimulation, RTs were maximally facilitated by emotionally congruent pairs of target and masked faces, while the presence of emotionally incongruent pairs significantly delayed RTs.

After suppressing the activity in occipital areas (active cathodal tDCS over left O1 session), results of the target emotion (fear, happiness) by condition (congruent, incongruent, neutral) ANOVA showed a significant interaction between the two factors $\left(F_{(2,46)}=5.58, p=0.007\right)$. Post hoc comparisons showed a significant reduction of RTs in the condition where target happy faces were coupled with masked fearful faces (Hf, mean RT 510 $\mathrm{ms}$ ), compared with all remaining conditions (Ff, mean RT $520 \mathrm{~ms}$, $p=0.049$; Fh, mean RT $520 \mathrm{~ms}, p=0.05$; Hn, mean RT $527 \mathrm{~ms}, p=$ 0.001 ; Hh, mean RT $521 \mathrm{~ms}, p=0.036$ ) except the Fn condition (mean RT $516 \mathrm{~ms}, p=0.26$; Fig. 3). Main effects of target emotion $\left(F_{(1,23)}=0.02, p=0.88\right)$ and condition $\left(F_{(2,46)}=1.15, p=0.32\right)$ were not significant.

To reveal the specific effects of inhibiting left occipital areas and exclude any unspecific confounding effect of the stimulation procedure, data from the session where tDCS was applied over left O1 were sham-normalized: mean RTs recorded during the sham tDCS session were subtracted from mean RTs recorded during the tDCS over left O1 session for each subject, in each separate condition $(\Delta \mathrm{RT}=$ active cathodal $\mathrm{tDCS}$ over left $\mathrm{O} 1-$ sham tDCS), in accordance with procedures used in other studies (Romei et al., 2010, 2011). This index represents the difference between the performance after active tDCS and the performance in normal physiological state (sham tDCS). Negative $\Delta$ RT val-

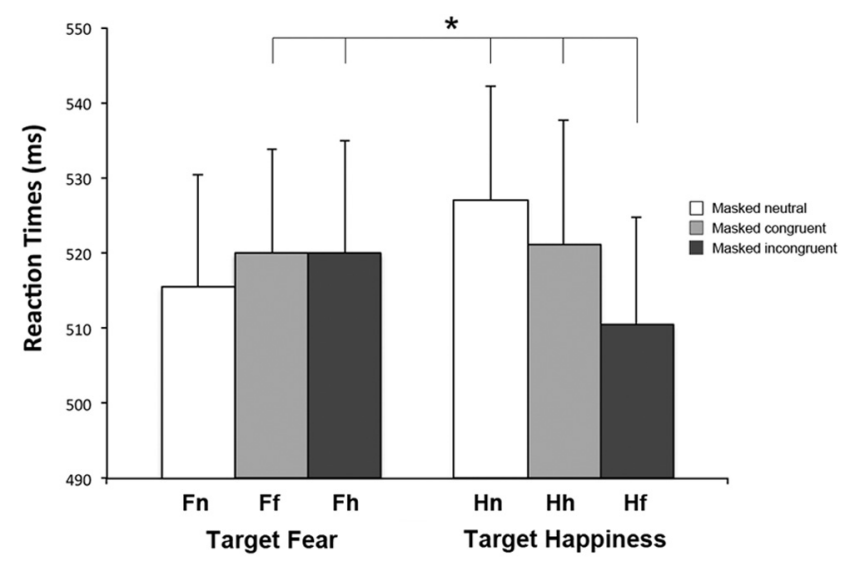

Figure 3. Experiment 1: Active tDCS over 01. Mean RTs are reported for each condition (neutral, congruent, incongruent) when the targets were fearful faces (columns on the left) and happy faces (columns on the right). Error bars indicate SEM. The asterisk indicates significantly faster RTs in response to a target happy face coupled with a masked fearful face ( $\mathrm{Hf}$ ) compared with $\mathrm{Ff}$, Fh, Hn, and Hh conditions (all $p s \leq 0.05$ ).

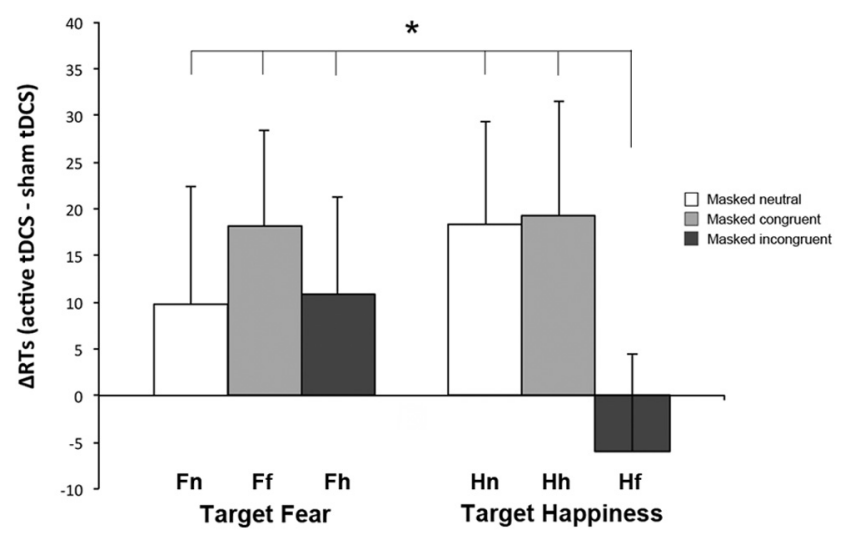

Figure 4. Experiment 1: Active tDCS over 01, sham-normalized data. Mean normalized RTs $(\Delta R T s)$ are reported for each condition (neutral, congruent, incongruent) when the targets were fearful faces (columns on the left) and happy faces (columns on the right). Error bars indicate SEM. The asterisk indicates significantly faster RTs in response to a target happy face coupled with a masked fearful face $(\mathrm{Hf})$ relative to all the remaining conditions ( $\mathrm{Fn}, \mathrm{Ff}, \mathrm{Fh}, \mathrm{Hn}$, and $\mathrm{Hh}$, all $p s<0.03$ ).

ues correspond to faster RTs. Normalized RTs ( $\Delta$ RTs) were entered in a $2 \times 3$ ANOVA with target emotion (fear, happiness) and condition (neutral, congruent, incongruent) as withinparticipants factors. A main effect of condition was evident $\left(F_{(2,46)}=6.51, p=0.003\right)$, while the main effect of target emotion was not significant $\left(F_{(1,23)}=0.08, p=0.78\right)$. More interestingly, and paralleling the results on raw data, the analysis highlighted a significant target emotion by condition interaction $\left(F_{(2,46)}=\right.$ 3.58, $p=0.036)$. Post hoc comparisons showed that during the session where tDCS was applied over left O1, participants were significantly faster when responding to a target happy face in the LFV coupled with a masked fearful face in the RVF (Hf condition, mean $\Delta \mathrm{RT}=-6 \mathrm{~ms})$, compared with all the remaining conditions (Fn, mean $\Delta \mathrm{RT}=10 \mathrm{~ms}, p=0.026 ; \mathrm{Ff}$, mean $\Delta \mathrm{RT}=18 \mathrm{~ms}$, $p=0.002 ; \mathrm{Fh}$, mean $\Delta \mathrm{RT}=11 \mathrm{~ms}, p=0.024 ; \mathrm{Hn}$, mean $\Delta \mathrm{RT}=$ $18 \mathrm{~ms}, p=0.002$; Hh, mean $\Delta \mathrm{RT}=19 \mathrm{~ms}, p=0.002$; Fig. 4 ). Together, these results show that, when the activity of occipital areas (O1) is suppressed, the RT facilitation for emotionally congruent pairs (either fearful or happy) of target and masked faces disappears and a specific facilitative effect is evident in the presence of masked fearful faces. 


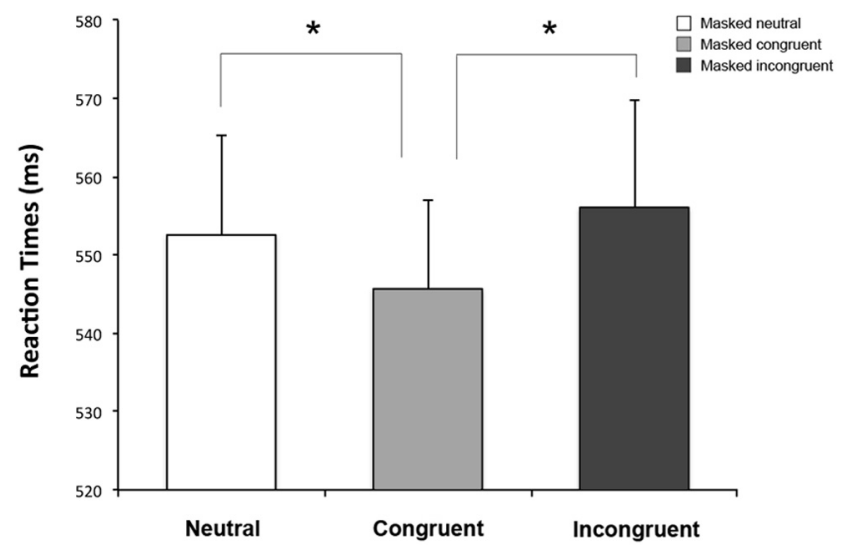

Figure 5. Experiment 2: Sham and active tDCS over Cz. Mean RTs are reported for the neutral condition (Fn, $\mathrm{Hn}$ ), the congruent condition ( $\mathrm{Ff}, \mathrm{Hh}$ ), and the incongruent condition (Fh, Hf). Error bars indicate SEM. Asterisks indicate significantly faster RTs in the congruent condition compared with the neutral and incongruent conditions (all $p s \leq 0.01$ ).

\section{Experiment 2: sham versus active cathodal tDCS over the vertex}

To ensure that the effects reported in Experiment 1 are specifically due to suppression of activity of $\mathrm{O} 1$, it is crucial to demonstrate that the inhibition of a different, nonvisual, area does not provide the same outcome. For this reason, in a control experiment, a group of healthy controls was tested with the same go/ no-go task used in Experiment 1 in two separate sessions; one after receiving sham and another after active cathodal tDCS over a control area, namely the vertex $(\mathrm{Cz})$. The session (active cathodal tDCS over Cz, sham tDCS) by target emotion (fear, happiness) by condition (congruent, incongruent, neutral) repeated-measures ANOVA revealed only a main effect of condition $\left(F_{(2,46)}=7.79, p=\right.$ 0.001). Post hoc comparisons showed faster RTs in the congruent conditions (Ff and Hh, mean RT $546 \mathrm{~ms}$ ) compared with both neutral (Fn and Hn, mean RT $553 \mathrm{~ms}, p=0.01$ ) and incongruent conditions (Fh and Hf, mean RT $556 \mathrm{~ms}, p=0.0005$ ), which did not differ statistically from each other $(p=0.2 ;$ Fig. 5$)$. Interestingly, no other main effect (session: $F_{(1,23)}=0.1, p=0.76$; emotion: $F_{(1,23)}=$ $3.35, p=0.08$ ) or interactions (all $F s<0.71$, all $p s>0.5$ ) were significant, suggesting that active cathodal tDCS over the vertex does not affect RTs compared with the sham stimulation. Overall, the control Experiment 2 revealed contrasting findings to Experiment 1 , because performances were similar between the sham session and the session where $\mathrm{tDCS}$ was applied over the vertex, i.e., a reduction of RTs with pairs of emotionally congruent target and masked faces. Notably, this pattern of results was similar to the participants' performance during the sham session of Experiment 1.

\section{Discussion}

Recent studies have revealed different patterns of implicit visual processing in patients with blindsight (de Gelder et al., 1999, 2001) and patients with hemianopia (Bertini et al., 2012). Therefore, since the cortical contribution to the implicit visual processing of emotional faces is still debated, the present study was designed to investigate this issue by using backward masking and active cathodal tDCS over the occipital cortex (Experiment 1), with stimulation at the vertex (Experiment 2) as a control site.

After sham stimulation over the occipital cortex in Experiment 1 , results revealed a reduction of RTs for consciously perceived emotional target faces ipsilateral to the stimulated site when coupled with emotionally congruent masked faces concurrently presented contralateral to the stimulated site. The effect was evident both for happy and fearful congruent couples of target and masked faces. This congruency-dependent effect suggests that when using backward masking, although the conscious discrimination of emotional faces is prevented, some implicit visual processing is still intact and this results in a response facilitation for pairs of emotionally congruent stimuli, in line with previous evidence testing healthy participants (Tamietto and de Gelder, 2008) and blindsight patients (de Gelder et al., 2001). However, after active cathodal tDCS over occipital sites (Experiment 1), the facilitative effect for emotionally congruent pairs of stimuli disappeared, revealing that the congruency effect for pairs of targets and emotional masked faces requires the contribution of the occipital visual cortex. Notably, the inhibition of the occipital cortex induced not only a disruption of the congruencydependent facilitation, but also revealed a significant reduction of response times when consciously perceived target happy faces, ipsilateral to the stimulated site, were coupled with masked fearful faces, contralateral to the stimulated site. This pattern of results is in line with previous evidence on hemianopic patients (Bertini et al., 2012). The control experiment (Experiment 2), where both sham and active cathodal tDCS were applied over the control site (vertex), revealed a reduction of RTs for congruent pairs of emotional stimuli, confirming the crucial role of the occipital cortex in mediating the congruency-dependent facilitation on response times.

The facilitation for pairs of emotionally congruent stimuli, observed both during the sham sessions (Experiment 1 and 2) and the session where the vertex was inhibited, suggests that the backward masked stimuli were visually processed in the absence of any conscious perception. Indeed, backward masking is known to affect visual processing by leaving intact the feedforward sweep of visual information, but interrupting the re-entrant feedback projections from the higher visual areas to the lower visual areas (Lamme and Roelfsema, 2000), thus preventing conscious processing. The processing of masked emotional signals has been shown to require the contribution of the subcortical low road pathway, as suggested by neuroimaging studies, which have shown specific patterns of activation in the amygdala in response to masked emotional faces. Amygdala activation increases during the presentation of subliminal fearful faces (Whalen et al., 1998; Liddell et al., 2005) or faces expressing negative emotions (Morris et al., 1999), while decreased blood oxygenation level-dependent signal intensity is observed in response to masked happy faces (Whalen et al., 1998).

However, the observation, in the present study, that the congruency-dependent facilitation disappeared after the inhibition of the occipital cortex, suggests that this effect might rely not only on the activity of the low road circuit, but also on the contribution of the occipital cortex and, possibly, of other visual areas of the cortical pathway. Interestingly, the lack of facilitation for congruent pairs of emotional stimuli following inhibition of the occipital cortex is in line with the performance of patients with hemianopia due to lesions or deafferentation of the occipital cortex (Bertini et al., 2012), but in contrast with performances observed in blindsight patients, when tested with similar go/ no-go tasks with emotional faces (de Gelder et al., 2001). Indeed, the blindsight patient G.Y. showed a reduction in response times when presented with bilateral congruent pairs of sad or fearful faces, compared with unilateral or bilateral incongruent pairs (de Gelder et al., 2001). In addition, blindsight patients also demonstrated above-chance performances in two alternative forcedchoice tasks requiring discrimination of emotional stimuli (de Gelder et al., 1999), the gender of neutral faces (Morris et al., 
2001), or the color of simple visual stimuli (Brent et al., 1994) and residual visual functions for several other visual features, such as spatial location, object orientation, and movement (for review, see Weiskrantz, 1986, 2001; Cowey, 2010). This pattern of results suggest that although blindsight patients report lesions to the primary visual cortex, their residual visual abilities might be mediated not only by the activity of the subcortical low road visual pathway, but also by the sparing of some cortical visual areas. In line with this hypothesis, sustained hemodynamic responses were found in the lateral occipital cortex and in the posterior fusiform gyrus of blindsight patients, during the presentation of images of natural objects (Goebel et al., 2001).

In contrast, the present results showed that when the activity of the occipital cortex was suppressed, the congruencydependent facilitation disappeared and a specific response facilitation for pairs of target happy faces and masked fearful faces became evident. Interestingly, this fear-specific facilitative effect is in line with the results of a recent behavioral study on hemianopic patients (Bertini et al., 2012), where unseen fearful faces facilitated behavioral responses to stimuli presented in the intact field, both during emotional and nonemotional tasks. These results suggest that when the occipital cortex is damaged or inhibited, and the visual processing for emotional faces is mainly dependent on the activation of the low road subcortical route, fearful faces represent the only stimuli visually processed and capable of mediating a behavioral response.

Notably, the response facilitation was observed only in the presence of masked fearful faces, coupled with target happy faces, while no facilitative effect was evident with congruent pairs of target and masked fearful faces. This pattern of results suggests that the presentation of consciously perceived (unmasked) fearful faces in the field ipsilateral to the stimulated site might have elicited inhibitory modulation of the implicit visual processing for masked fearful stimuli, possibly mediated by the low road subcortical circuit. In line with this hypothesis, it has been observed that conscious fear perception elicits negative functional connectivity within visual pathways to the amygdala (Williams et al., 2006). This finding suggests the possibility of a dynamic interplay between the high road and the low road, which is also supported by the evidence that the two pathways do not exhibit a complete anatomical segregation (Williams et al., 2006). Indeed, the amygdala has a crucial role in the subcortical pathway, but is also a pivotal structure in perceiving and recognizing conscious fearful signals, most notably, fearful faces (Adolphs et al., 1994, 1995; Morris et al., 1996). In addition, the pulvinar is a subcortical structure, which is not only connected with many other subcortical sites, but also shows reciprocal connections throughout the entire cortex and contributes to conscious fear recognition (Ward et al., 2005, 2007).

Alternatively, the observation of a facilitative effect only in the presence of pairs of target happy faces and masked fearful faces might be explained by a greater activation of the subcortical pathway in ambiguous and uncertain environmental circumstances, consistent with recent evidence showing a greater activation of the amygdala in the presence of ambiguous threat (Adams et al., 2003) or during an unpredictable series of auditory tones (Herry et al., 2007).

Overall, the results of the present study disentangle the contribution of the occipital cortex in the implicit visual processing of emotional faces. In particular, the occipital cortex seems to play a crucial role in mediating high-order implicit visual processes, such as the congruency-dependent facilitative effects. In contrast, the fear-specific facilitation observed after inhibition of the occipital cortex might be mainly mediated by the activation of the subcortical pathway. Although the existence of a subcortical pathway for emotional visual processing has been debated (for review, see Pessoa and Adolphs, 2010), evidence for the anatomical substrate of the low-road subcortical circuit has been provided by animal studies, reporting reciprocal connections between structures involved in emotional and implicit visual processing such as the superior colliculus, the thalamus, and the amygdala (Jones and Burton, 1976; Romanski et al., 1997; Linke et al., 1999; Shi and Davis, 2001). In addition, other recent studies have demonstrated the existence of a direct visual pathway encompassing these neural sites, both in animals (Chomsung et al., 2008; Day-Brown et al., 2010) and in humans (Tamietto et al., 2012). Due to the crucial involvement of the amygdala, this neural circuit is preferentially activated by fear-related signals and, therefore, might have a great relevance for survival, with consequent evolutionary advantage. Indeed, this pathway, showing an automatic and fast activation (Luo et al., 2010), is presumably involved in implementing adaptive defensive behaviors (de Gelder et al., 2004).

The present findings also shed light on the performances of blindsight and hemianopic patients, suggesting that their different behavioral results might be ascribed to different lesional profiles. Patients described in classical blindsight studies demonstrate high-order visual processing, similar to the performance of healthy participants where the conscious visual processing was prevented by backward masking. This finding suggests that their performances might rely on a peculiar functional and anatomical reorganization of the visual system, allowing first for contributions from the subcortical low road pathway, but also from the high road cortical circuit, by way of some spared occipital areas. In contrast, the performance of hemianopic patients reveals a fear-specific effect, similar to the facilitation observed in healthy participants when the conscious visual processing was prevented by backward masking and the occipital cortex was inhibited by tDCS, and might reflect an automatic, low-order process, mainly dependent upon the activation of the low road subcortical circuit.

\section{References}

Adams RB Jr, Gordon HL, Baird AA, Ambady N, Kleck RE (2003) Effects of gaze on amygdala sensitivity to anger and fear faces. Science 300:1536. CrossRef Medline

Adolphs R (2002) Recognizing emotion from facial expressions: psychological and neurological mechanisms. Behav Cogn Neurosci Rev 1:21-62. CrossRef Medline

Adolphs R, Tranel D, Damasio H, Damasio A (1994) Impaired recognition of emotion in facial expressions following bilateral damage to the human amygdala. Nature 372:669-672. CrossRef Medline

Adolphs R, Tranel D, Damasio H, Damasio AR (1995) Fear and the human amygdala. J Neurosci 15:5879-5891. Medline

Antal A, Nitsche MA, Paulus W (2001) External modulation of visual perception in humans. Neuroreport 12:3553-3555. CrossRef Medline

Antal A, Kincses TZ, Nitsche MA, Paulus W (2003a) Manipulation of phosphene thresholds by transcranial direct current stimulation in man. Exp Brain Res 150:375-378. Medline

Antal A, Kincses TZ, Nitsche MA, Paulus W (2003b) Modulation of moving phosphene thresholds by transcranial direct current stimulation of V1 in human. Neuropsychologia 41:1802-1807. CrossRef Medline

Antal A, Kincses TZ, Nitsche MA, Bartfai O, Paulus W (2004a) Excitability changes induced in the human primary visual cortex by transcranial direct current stimulation: direct electrophysiological evidence. Invest Ophthalmol Vis Sci 45:702-707. CrossRef Medline

Antal A, Varga ET, Nitsche MA, Chadaide Z, Paulus W, Kovacs G, Vidnyánszky Z (2004b) Direct current stimulation over MT+/V5 modulates motion aftereffect in humans. Neuroreport 15:2491-2494. CrossRef Medline 
Bertini C, Leo F, Avenanti A, Làdavas E (2010) Independent mechanisms for ventriloquism and multisensory integration as revealed by theta-burst stimulation. Eur J Neurosci 31:1791-1799. CrossRef Medline

Bertini C, Cecere R, Làdavas E (2012) I am blind, but I "see" fear. Cortex. Advance online publication. Retrieved March 3, 2012. doi:10.1016/ j.cortex.2012.02.006. CrossRef

Brent PJ, Kennard C, Ruddock KH (1994) Residual colour vision in a human hemianope: spectral responses and colour discrimination. Proc Biol Sci 256:219-225. CrossRef Medline

Chomsung RD, Petry HM, Bickford ME (2008) Ultrastructural examination of diffuse and specific tectopulvinar projections in the tree shrew. J Comp Neurol 510:24-46. CrossRef Medline

Cowey A (2010) The blindsight saga. Exp Brain Res 200:3-24. CrossRef Medline

Day-Brown JD, Wei H, Chomsung RD, Petry HM, Bickford ME (2010) Pulvinar projections to the striatum and amygdala in the tree shrew. Front Neuroanat 4:143. Medline

de Gelder B, Vroomen J, Pourtois G, Weiskrantz L (1999) Non-conscious recognition of affect in the absence of striate cortex. Neuroreport 10: 3759-3763. CrossRef Medline

de Gelder B, Pourtois G, van Raamsdonk M, Vroomen J, Weiskrantz L (2001) Unseen stimuli modulate conscious visual experience: evidence from inter-hemispheric summation. Neuroreport 12:385-391. CrossRef Medline

de Gelder B, Snyder J, Greve D, Gerard G, Hadjikhani N (2004) Fear fosters flight: a mechanism for fear contagion when perceiving emotion expressed by a whole body. Proc Natl Acad Sci U S A 101:16701-16706. CrossRef Medline

Ekman P, Friesen W (1976) Pictures of Facial Affect. Palo Alto, CA: Consulting Psychologists.

Esteves F, Ohman A (1993) Masking the face: recognition of emotional facial expressions as a function of the parameters of backward masking. Scand J Psychol 34:1-18. CrossRef Medline

Etkin A, Klemenhagen KC, Dudman JT, Rogan MT, Hen R, Kandel ER, Hirsch J (2004) Individual differences in trait anxiety predict the response of the basolateral amygdala to unconsciously processed fearful faces. Neuron 44:1043-1055. CrossRef Medline

Goebel R, Muckli L, Zanella FE, Singer W, Stoerig P (2001) Sustained extrastriate cortical activation without visual awareness revealed by fMRI studies of hemianopic patients. Vision Res 41:1459-1474. CrossRef Medline

Herry C, Bach DR, Esposito F, Di Salle F, Perrig WJ, Scheffler K, Lüthi A, Seifritz E (2007) Processing of temporal unpredictability in human and animal amygdala. J Neurosci 27:5958-5966. CrossRef Medline

Jones EG, Burton H (1976) A projection from the medial pulvinar to the amygdala in primates. Brain Res 104:142-147. CrossRef Medline

Lamme VA, Roelfsema PR (2000) The distinct modes of vision offered by feedforward and recurrent processing. Trends Neurosci 23:571-579. CrossRef Medline

LeDoux J (1996) The emotional brain: the mysterious underpinnings of emotional life. New York: Simon and Schuster.

Liddell BJ, Brown KJ, Kemp AH, Barton MJ, Das P, Peduto A, Gordon E, Williams LM (2005) A direct brainstem-amygdala-cortical 'alarm' system for subliminal signals of fear. Neuroimage 24:235-243. CrossRef Medline

Linke R, De Lima AD, Schwegler H, Pape HC (1999) Direct synaptic connections of axons from superior colliculus with identified thalamo- amygdaloid projection neurons in the rat: possible substrates of a subcortical visual pathway to the amygdala. J Comp Neurol 403:158-170. CrossRef Medline

Luo Q, Holroyd T, Majestic C, Cheng X, Schechter J, Blair RJ (2010) Emotional automaticity is a matter of timing. J Neurosci 30:5825-5829. CrossRef Medline

Morris JS, Frith CD, Perrett DI, Rowland D, Young AW, Calder AJ, Dolan RJ (1996) A differential neural response in the human amygdala to fearful and happy facial expressions. Nature 383:812-815. CrossRef Medline

Morris JS, Ohman A, Dolan RJ (1998) Conscious and unconscious emotional learning in the human amygdala. Nature 393:467-470. CrossRef Medline

Morris JS, Ohman A, Dolan RJ (1999) A subcortical pathway to the right amygdala mediating "unseen" fear. Proc Natl Acad Sci U S A 96:16801685. CrossRef Medline

Morris JS, DeGelder B, Weiskrantz L, Dolan RJ (2001) Differential extrageniculostriate and amygdala responses to presentation of emotional faces in a cortically blind field. Brain 124:1241-1252. CrossRef Medline

Pessoa L, Adolphs R (2010) Emotion processing and the amygdala: from a 'low road' to 'many roads' of evaluating biological significance. Nat Rev Neurosci 11:773-783. CrossRef Medline

Romanski LM, Giguere M, Bates JF, Goldman-Rakic PS (1997) Topographic organization of medial pulvinar connections with the prefrontal cortex in the rhesus monkey. J Comp Neurol 379:313-332. CrossRef Medline

Romei V, Gross J, Thut G (2010) On the role of prestimulus alpha rhythms over occipito-parietal areas in visual input regulation: correlation or causation? J Neurosci 30:8692-8697. CrossRef Medline

Romei V, Driver J, Schyns PG, Thut G (2011) Rhythmic TMS over parietal cortex links distinct brain frequencies to global versus local visual processing. Curr Biol 21:334-337. CrossRef Medline

Shi C, Davis M (2001) Visual pathways involved in fear conditioning measured with fear-potentiated startle: behavioral and anatomic studies. J Neurosci 21:9844-9855. Medline

Tamietto M, de Gelder B (2008) Affective blindsight in the intact brain: neural interhemispheric summation for unseen fearful expressions. Neuropsychologia 46:820-828. CrossRef Medline

Tamietto M, Pullens P, de Gelder B, Weiskrantz L, Goebel R (2012) Subcortical connections to human amygdala and changes following destruction of the visual cortex. Curr Biol 22:1449-1455. CrossRef Medline

Ward R, Danziger S, Bamford S (2005) Response to visual threat following damage to the pulvinar. Curr Biol 15:571-573. CrossRef Medline

Ward R, Calder AJ, Parker M, Arend I (2007) Emotion recognition following human pulvinar damage. Neuropsychologia 45:1973-1978. CrossRef Medline

Weiskrantz L (1986) Blindsight: a case study and implications. Oxford: Oxford UP.

Weiskrantz L (2001) Blindsight. In: Handbook of neuropsychology: disorders of visual behavior Vol 4. (Behrmann M, ed), pp 215-237. Amsterdam: Elsevier.

Whalen PJ, Rauch SL, Etcoff NL, McInerney SC, Lee MB, Jenike MA (1998) Masked presentations of emotional facial expressions modulate amygdala activity without explicit knowledge. J Neurosci 18:411-418. Medline

Williams LM, Das P, Liddell BJ, Kemp AH, Rennie CJ, Gordon E (2006) Mode of functional connectivity in amygdala pathways dissociates level of awareness for signals of fear. J Neurosci 26:9264-9271. CrossRef Medline 